\title{
Mechanical properties of a bacterial cellulose/ polyethylene glycol gel with a peripheral region crosslinked by polyethylene glycol diacrylate
}

\author{
Yukari Numata $^{1,2}$, Shiro Masaki ${ }^{2}$ and Kenji Tajima ${ }^{3}$ \\ Polymer Journal (2016) 48, 317-321; doi:10.1038/pj.2015.104; published online 4 November 2015
}

\section{INTRODUCTION}

Bacterial cellulose (BC) is a unique type of three-dimensionally structured biopolymer gel based on the ultrafine fibers of pure cellulose produced by Acetobacter ${ }^{1-3}$ that are several hundred times thinner than the cellulose fibers of plants. This feature gives BC gels a unique combination of softness, translucence, biocompatibility and water-retention ability, ${ }^{4-6}$ because of which they are widely studied as potential soft materials for biomedical applications. ${ }^{7-10}$ However, they have a significant drawback in such applications: the large amount of water in the gels can be easily expelled under even slight pressure, thus causing them to dry up easily. In an attempt to overcome this problem, nonvolatile and thermo-responsive BC gels were developed by swelling them in a nonvolatile solvent (polyethylene glycol (PEG)) rather than water. The resulting gels, which are denoted as BC/PEG, ${ }^{11}$ are opaque and solid at low temperature, but change to a transparent and elastic state at high temperature. A BC/PEG gel prepared from equal amounts of PEGs with molecular weights (Mw) of 200 and 1000 was found to become transparent and elastic as the temperature increased above $25^{\circ} \mathrm{C} .{ }^{12}$ As this change depends on the melting point of PEG, the temperature of the thermo-responsiveness can be controlled by selecting an appropriate $\mathrm{Mw}$ of PEG. These BC/PEG gels were subsequently used to create an overall composite gel with polyethylene glycol diacrylate (PEGDA) in order to improve its mechanical properties under compression; the resulting gels being denoted as BC/PEG-PEGDA ${ }^{12}$ based on the fact that PEG does not bind to the $\mathrm{BC}$ by a strong covalent bond. The thermo-responsiveness of these BC/PEG-PEGDA gels is maintained as they change from opaque and solid to transparent and elastic, but their increased strength under compression makes them hard and brittle. Thus, they are rendered unsuitable for use as a soft gel material for skin in medical and cosmetic applications, where both a suitable hardness and elongation are required.

To address the limitations of BC/PEG-PEGDA gel, this study explores the possibility of developing a BC/PEG gel with only its peripheral region crosslinked by PEGDA. The mechanical properties and hardness of such a gel are herein discussed from the point of view of its potential application as a soft material in the fields of medicine and cosmetics. In addition, we focus on the thermo-responsiveness of these novel BC/PEG-PEGDA gels with regard to their change from opaque to transparent.

\section{MATERIALS AND METHODS}

The BC hydrogel was prepared using Gluconacetobacter xylinus ATCC53582 in Hestrin and Schramm's medium at $28^{\circ} \mathrm{C}$ for 3 days under static conditions. ${ }^{13}$ The $\mathrm{BC}$ gel formed was then treated with $1 \% \mathrm{NaOH}$ aqueous solution and washed with distilled water. The procedure for preparing a BC/PEG gel with its peripheral region crosslinked by PEGDA is shown in Supplementary Figure S1 (method A). Here, equal weights of PEG (Kishida Chemical Co., Ltd, Osaka, Japan) with Mw of 200 and 1000 (PEG 200 and PEG 1000, respectively) were mixed to create PEG $200-1000$ in a liquid state that was then used to prepare swollen BC gels in accordance with a previously described method. ${ }^{11}$ For this, purified BC hydrogels were added to the PEG 200-1000 solution diluted $90 \%$ (v/v) in distilled water, and then heated for $90 \mathrm{~min}$ at $110^{\circ} \mathrm{C}$. Next, the surface of the gels was washed with distilled water to remove any excess PEG, after which they were dried at $65^{\circ} \mathrm{C}$ in air on a glass plate. Introduction of PEGDA (A-400: polyethylene Glycol 400 Diacrylate, $n=9$, Shin-Nakamura Chemical Co., Ltd, Wakayama, Japan) to the BC/PEG gels was also achieved using a modified variant of a previously described method. The procedure for preparing a $\mathrm{BC} / \mathrm{PEG}$ gel crosslinked by PEGDA ${ }^{12}$ is shown in Supplementary Figure S1 (method B). With this, BC/PEG gels were immersed in PEGDA supplemented with $0.1 \%$ 1-hydroxycyclohexyl phenyl ketone (Sigma-Aldrich Co., St Louis, MO, USA) as an initiator and diluted by 40 or $80 \%$ PEG 200 1000. These were then incubated for 2,4 or $24 \mathrm{~h}$ at $35^{\circ} \mathrm{C}$ in the dark, and the resulting PEGDA-containing BC/PEG gels were kept on the glass plate and irradiated by ultraviolet light $(365 \mathrm{~nm})$ for $90 \mathrm{~s}$ at $5 \mathrm{~mW} \mathrm{~cm}^{-2}$ at room temperature. The gels obtained were washed and dried using the same procedure described above, and then cut into a disk or dumb-bell shape.

The structures of the disk-shaped BC/PEG-PEGDA and BC/PEG gels were observed using a JSM-7001FA scanning electron microscope (JEOL Co., Tokyo, Japan) operating at an accelerating voltage of $10 \mathrm{kV}$ after first removing PEG and freeze drying. All samples were coated with carbon to a thickness of $\sim 20 \mathrm{~nm}$ using a VC-100 W carbon coater (Vacuum Device Inc., Mito, Japan). Vertical sections of each sample were observed by tilting up the sample stage to $70^{\circ}$.

${ }^{1}$ General Education, Faculty of Commerce, Otaru University of Commerce, Otaru, Japan; ${ }^{2}$ Department of Materials Chemistry, Asahikawa National College of Technology, Asahikawa, Japan and ${ }^{3}$ Division of Applied Chemistry, Faculty of Engineering, Hokkaido University, Sapporo, Japan

Correspondence: Professor Y Numata, General Education, Faculty of Commerce, Otaru University of Commerce, 3-5-21, Midori, Otaru 047-8501, Japan.

E-mail: numata@res.otaru-uc.ac.jp

Received 29 June 2015; revised 27 September 2015; accepted 28 September 2015; published online 4 November 2015 
The weight ratio of crosslinked PEGDA $\left(W_{P E G D A}\right)$ in the BC/PEG-PEGDA gels was determined using a previously described method. ${ }^{12}$ Accordingly, PEG was removed from the disk-shaped gel samples by immersing them in distilled water for 1 week at $35^{\circ} \mathrm{C}$. The weight of the $\mathrm{BC}$ fiber was considered negligible relative to that of PEG and crosslinked PEGDA, as the BC fibers make up $<1 \%$ of the BC/PEG gel. ${ }^{11}$ After drying, the values were determined using the equation:

$$
W_{P E G D A}=W_{\text {residue }} / W_{0}
$$

where $W_{\text {residue }}$ is the weight of gel after PEG removal and drying, and $W_{0}$ is the weight of the initial BC/PEG-PEGDA gel.

Tensile strength testing was performed at $35^{\circ} \mathrm{C}$ using a Tensilon RTG- 1225 tensile-compressive tester (Orientec Co., Tokyo, Japan). For this, the samples were cut into a dumb-bell shape (Japanese Industrial Standards (JIS) K6251 dumb-bell test pieces-7) and pulled parallel to the BC layers at a strain rate of $2 \mathrm{~mm} \mathrm{~min}{ }^{-1}$; then, the failure point in each tensile test was determined from the peak of the stress-strain curve generated. Hardness was measured using a Type OO Teclock durometer (GS-754G, Teclock Co., Okaya, Japan) attached to a Type GS-610 stand (Teclock Co.). Pressure was measured at 500 gf using a lowering speed of $5 \mathrm{~mm} \mathrm{~s}^{-1}$. Statistical analysis was carried out based on a $t$-test, from which it was judged that the difference was significant when $P \leqslant 0.05$.

The optical transmission of each sample at a wavelength of $600 \mathrm{~nm}$ was measured using a V-630iRM spectrometer (JASCO Co., Tokyo, Japan) equipped with an HMC-711 (JASCO Co.) constant-temperature microcell holder. This was achieved by fitting the samples into a glass cell, which was held in place by a glass plate and set inside the microcell holder. This holder was then set to temperatures between 15 and $35^{\circ} \mathrm{C}$ at intervals of $5{ }^{\circ} \mathrm{C}$.

\section{RESULTS AND DISCUSSION}

Scanning electron microscope images of the surface and vertical section of the BC/PEG and BC/PEG-PEGDA gels are presented in Figure 1, revealing a clear network of cellulose nanofibers at the surface of the BC/PEG gel (Figure 1a). A very different surface morphology was observed in the case of the BC/PEG-PEGDA gels prepared by immersion for $2 \mathrm{~h}$ in PEGDA concentrations of 40 or $80 \%$ (Figures $1 \mathrm{~b}$ and $\mathrm{c}$ ), in that the network structure completely disappeared. This suggests that PEGDA in contact with the surface of the gel formed a composite structure with BC/PEG, with an increase in PEGDA concentration or immersion time resulting in a denser composite structure (Figure 1d). The vertical sections of the BC/PEGPEGDA gels treated with 40\% PEGDA for $2 \mathrm{~h}$ reveal that most of the cellulose fibers near the surface are covered by aggregations of crosslinked PEGDA (Figure 1e), whereas at greater depths, crosslinked PEGDA is located between the cellulose fibers (Figure 1f). This same effect is seen with the gel prepared using 40\% PEGDA for $4 \mathrm{~h}$ and $80 \%$ PEGDA for 2 and $4 \mathrm{~h}$, indicating that a concentration gradient of crosslinked PEGDA is created within the gel.

To determine the extent to which the diffusion of PEGDA responsible for creating a concentration gradient is affected by the rate at which it is introduced, the weight of crosslinked PEGDA was measured in each sample. Figure 2 shows the weight of crosslinked PEGDA polymer in the BC/PEG-PEGDA, with both samples reaching the initial PEGDA concentration after $24 \mathrm{~h}$. This result supports the
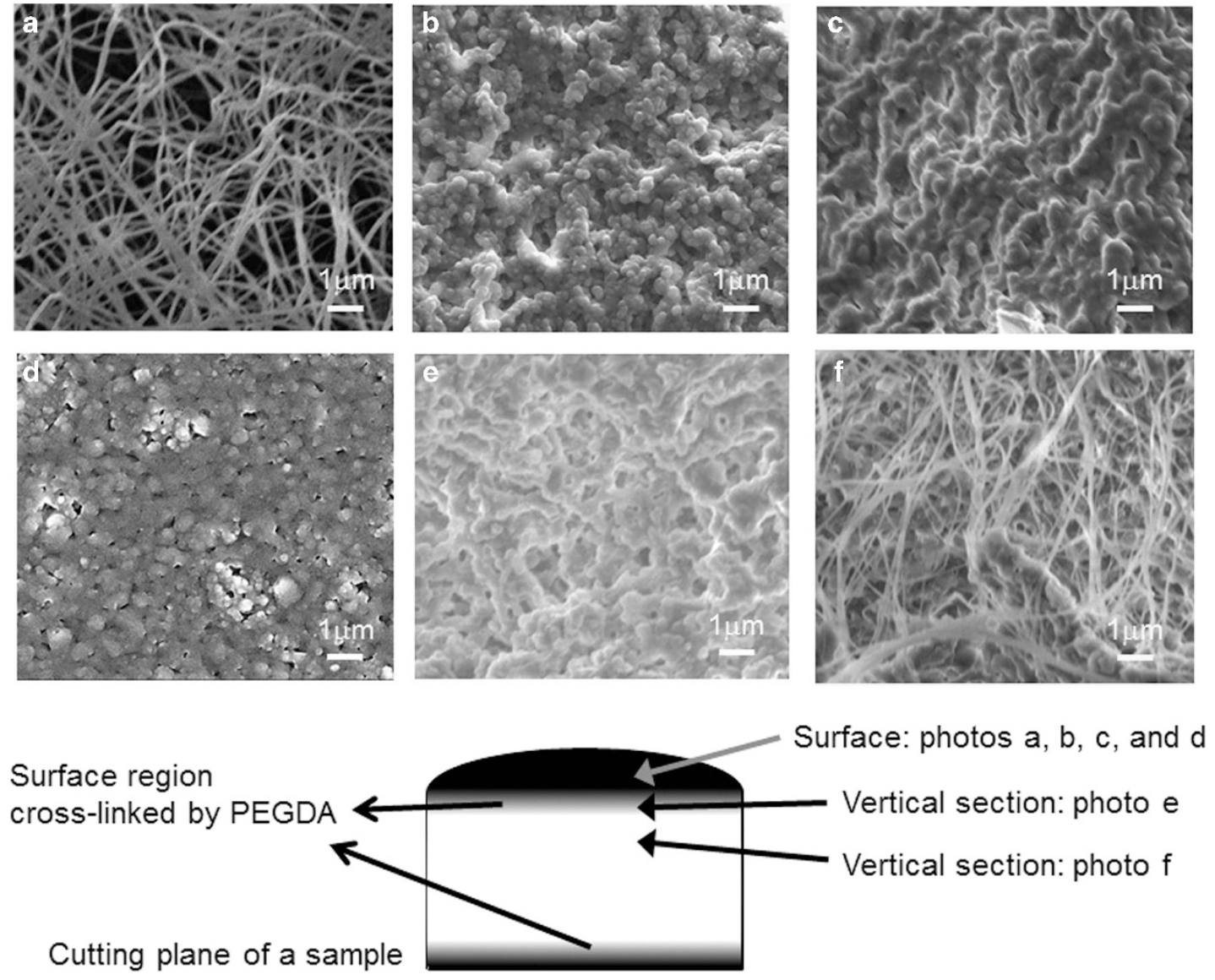

Figure 1 Scanning electron microscope (SEM) images showing the surface of (a) BC/PEG gel, (b) BC/PEG-40\% PEGDA_2 h, (c) BC/PEG-80\% PEGDA_2h and (d) BC/PEG-80\% PEGDA_24 $\mathrm{h}$; vertical sections of (e) the near surface region of BC/PEG-40\% PEGDA_2 $\mathrm{h}$ and (f) the near fiber phase of BC/PEG-40\% PEGDA_2 $\mathrm{h}$. In all samples, PEG was removed by washing before freeze drying. Vertical sectional images were obtained by tilting up the sample stage to $70^{\circ}$. 
notion that a crosslinked PEGDA concentration gradient is present in the gels treated for 2 and $4 \mathrm{~h}$, with the PEGDA clearly entering the $\mathrm{BC} / \mathrm{PEG}$ gel through its surface, until eventually the PEGDA concentration in the gel equals the initial PEGDA concentration. In other words, when a BC/PEG gel is immersed for a sufficient time in PEG and PEGDA supplemented with an initiator, the crosslinked PEGDA concentration in the gel reaches equilibrium. If, however, the BC/PEG gel is immersed for a shorter period of time, then a crosslinked PEGDA concentration gradient is created.

Figure 3 shows the relation between the hardness of the composite gel and its tensile fracture stress/strain, revealing that treatment with PEGDA increases the hardness over the nontreated BC/PEG gel; that is, the material's hardness is related to its deformation resistance. Moreover, although the samples prepared with 80\% PEGDA exhibited an increase in hardness with treatment time, the hardness of the samples prepared with $40 \%$ PEGDA remained fairly consistent at $\sim 820-875 \mathrm{mN}$. No significant difference was found in the hardness of the gels treated for 4 and $24 \mathrm{~h}$ based on the results of the $t$-test.

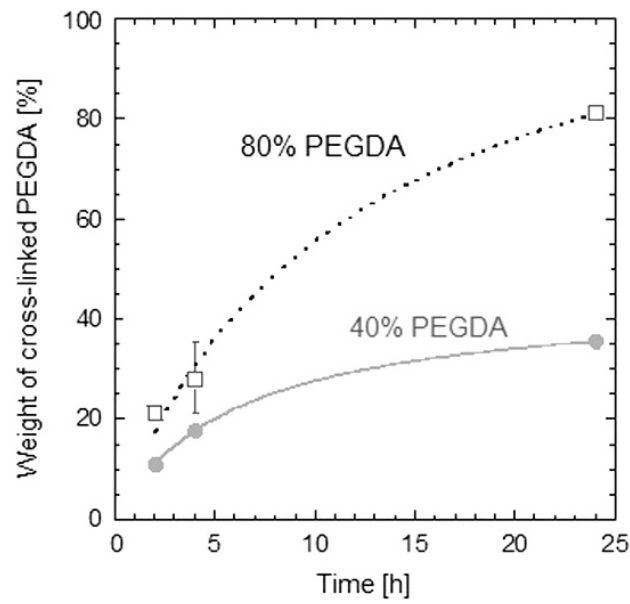

Figure 2 Dependence of PEGDA impregnation time and introduction rate on the weight of crosslinked PEGDA polymer dried on BC/PEG-PEGDA. The PEG was completely removed from the BC/PEG-PEGDA samples by washing and all results represent the mean values \pm s.d. $(n=3)$.

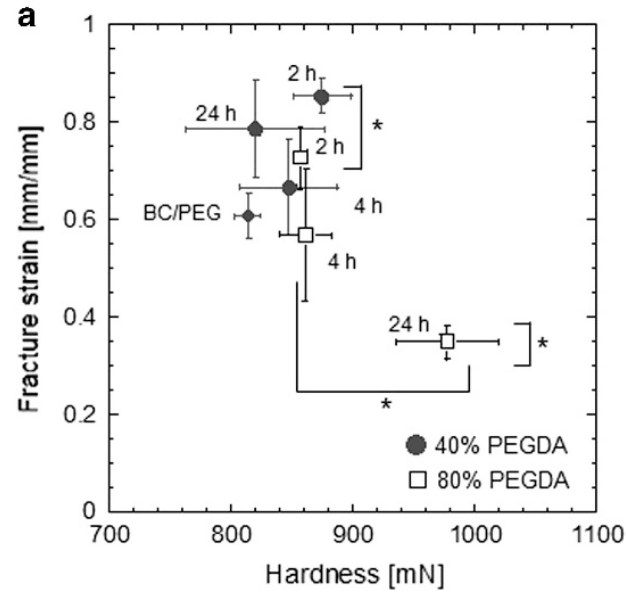

These results can be explained by the fact that the samples prepared with 40\% PEGDA include at least 60\% PEG that decreases the deformation resistance. The samples prepared with 80\% PEGDA, on the other hand, become predominantly crosslinked PEGDA with increasing treatment time. For example, the sample treated for $4 \mathrm{~h}$ included $\sim 30 \%$ crosslinked PEGDA (Figure 2 ) and had a hardness similar to the $40 \%$ PEGDA sample, but the $80 \%$ PEGDA $24 \mathrm{~h}$ sample became much harder than the others. This result indicates that the weight of crosslinked PEGDA in the gels directly affects their hardness, and that this difference extends to the fracture strain. Consequently, there is a correlation between hardness and fracture strain in the case of the $80 \%$ PEGDA samples that is not seen in the $40 \%$ samples. Given that the tensile properties of $\mathrm{BC}$ composite materials are influenced by the strong hydrogen bonds between cellulose fibers, the loss of the original $\mathrm{BC}$ network structure from the external phase (that is, PEG and crosslinked PEGDA) tends to weaken them ${ }^{14-16}$ and allow the fibers to extend further during stretching. In BC/PEG gel, this has the effect of increasing the fracture strain $\left(0.62 \mathrm{~mm} \mathrm{~mm}^{-1}\right)$ and reducing the fracture stress $(0.88 \mathrm{MPa})$ relative to $\mathrm{BC}$ hydrogel alone $\left(0.4 \mathrm{~mm} \mathrm{~mm}^{-1}, 2.2 \mathrm{MPa}\right)$. However, the fracture strain and stress of the gel prepared with $40 \%$ PEGDA for $2 \mathrm{~h}\left(0.86 \mathrm{~mm} \mathrm{~mm}^{-1}\right.$, $0.94 \mathrm{MPa})$ were notably larger than that of the $4 \mathrm{~h}\left(0.64 \mathrm{~mm} \mathrm{~mm}^{-1}\right.$, $0.58 \mathrm{MPa})$ and $24 \mathrm{~h}\left(0.83 \mathrm{~mm} \mathrm{~mm}^{-1}, 0.70 \mathrm{MPa}\right)$ samples. The fracture strain of the $4 \mathrm{~h}$ sample, and the fracture stress of the 2 and $24 \mathrm{~h}$ samples, showed no significant deviation from that of BC/PEG. In addition, the fracture strain of the gel prepared with $80 \%$ PEGDA decreased with increasing treatment time, whereas the fracture stress of the $2 \mathrm{~h}\left(0.76 \mathrm{~mm} \mathrm{~mm}^{-1}, 0.83 \mathrm{MPa}\right)$ and $24 \mathrm{~h}\left(0.35 \mathrm{~mm} \mathrm{~mm}^{-1}\right.$, $0.82 \mathrm{MPa}$ ) samples were essentially equal in value and showed no significant deviation from that of BC/PEG. As the composite gel prepared with $80 \%$ PEGDA for $24 \mathrm{~h}$ was predominantly crosslinked PEGDA polymer, it was very brittle under elongation. In contrast, with the 40\% PEGDA and 80\% PEGDA samples treated for 2 and $4 \mathrm{~h}$, the fracture strain and stress value increased in the order 40\% PEGDA for $2 \mathrm{~h}, 80 \%$ PEGDA for $2 \mathrm{~h}, 40 \%$ PEGDA for $4 \mathrm{~h}$ and $80 \%$ PEGDA for $4 \mathrm{~h}$. In other words, this order is not directly related to the weight of crosslinked PEGDA, suggesting that the depth of the composite has a greater effect. For this reason, a treatment time of $2 \mathrm{~h}$ is considered optimal in terms of the final mechanical properties, as this facilitates

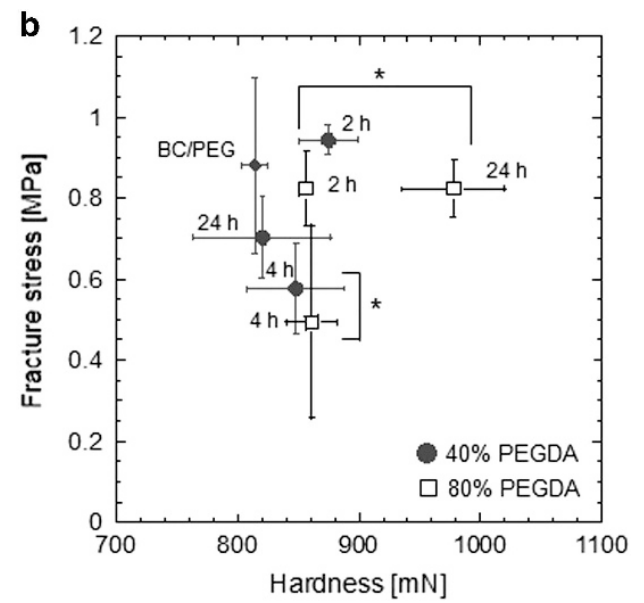

Figure 3 Relationship between (a) hardness and tensile fracture strain and (b) hardness and tensile fracture stress for BC/PEG-PEGDA. All results are shown as the mean values \pm s.d. $(n=5$ ). Hardness: $40 \% 2 \mathrm{~h}, 80 \% 2,4$ and $24 \mathrm{~h}$ are $* P \leqslant 0.05$ (for BC/PEG); fracture strain: $40 \% 2$ and $24 \mathrm{~h}, 80 \% 2$ and $24 \mathrm{~h}$ are ${ }^{*} P \leqslant 0.05$ (for $\mathrm{BC} / \mathrm{PEG}$ ); fracture stress: $40 \% 4 \mathrm{~h}$ and $80 \% 4 \mathrm{~h}$ are ${ }^{*} P \leqslant 0.05$ (for $\mathrm{BC} / \mathrm{PEG}$ ). 


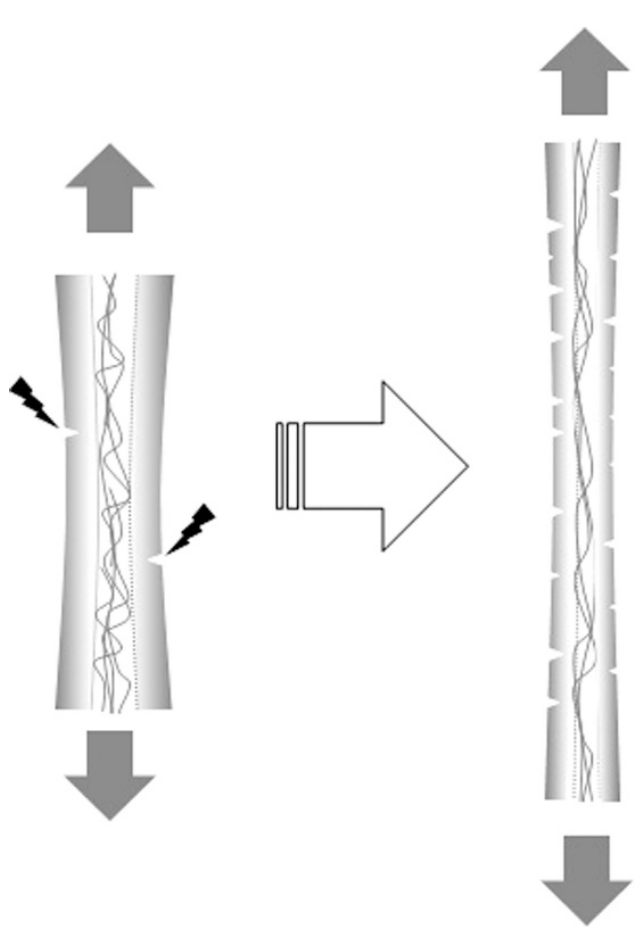

Figure 4 Schematic of the tensile testing of BC/PEG-PEGDA gel in which initial fracture of the surface composite is followed by the inner cellulose fiber part.

extension of the gel. Figure 4 shows a schematic of how this affects the gel sample during tensile testing, wherein the composite phase in the peripheral region is broken early on, but the fiber phase in the center remains. In other words, if a large amount of crosslinked PEGDA is present in the composite phase, it becomes brittle and is more easily broken. This in turn causes it to peel away from the gel early on, so that the fiber phase is unable to withstand elongation. As the retention of the fiber phase is vital to reducing the brittleness of the material, the concentration gradient of the PEGDA polymer is clearly a critical factor (Figure 1).

The mechanical properties of several cellulose composites have been reported by selective dissolution of the fiber surface, ${ }^{15,17-19}$ revealing the hydrogen bond between fibers to be an important factor. Thus, with cellulose sheets consisting of a micro-sized fiber network such as filter paper, larger voids and fewer hydrogen-bonded fiber-fiber interactions are formed. In a composite, these voids are filled by the matrix, leading to a strong interface and good stress transfer capability that lead to a marked increase in strength. However, as the BC sheet has a stronger nano-sized cellulose fiber network structure compared with that of a sheet prepared from microfibrillated cellulose, its mechanical properties decrease with any loss of continuity of the original network structure. ${ }^{15}$ In this study, the decrease in strength of the BC gel fully composited with crosslinked PEGDA was similar to that of a $\mathrm{BC}$ composite sheet, but the peripheral region crosslinked by an appropriate concentration of PEGDA meant that the fracture strain and fracture stress could be maintained or even improved. This is because the introduction of crosslinked PEGDAs between cellulose fibers allows them to move more easily, thus making the PEGDA concentration an important consideration in creating a PEGDA network structure. That is, a high concentration of PEGDA molecules results in more entanglement in the $\mathrm{BC} / \mathrm{PEG}$ gel, thereby increasing the number of crosslinked points. A composite gel produced with a

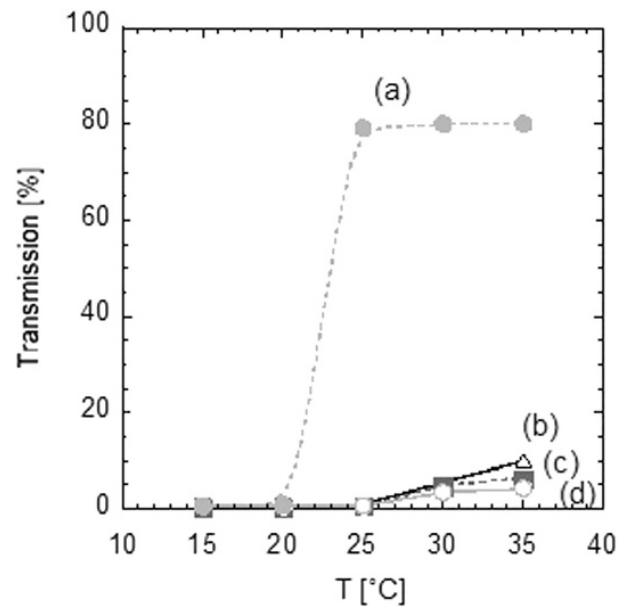

Figure 5 Transmission at $\lambda=600 \mathrm{~nm}$ of (a) BC/PEG and BC/PEG-40\% PEGDA (b) $2 \mathrm{~h}$, (c) $4 \mathrm{~h}$ and (d) $24 \mathrm{~h}$. Gel thickness was (a) 2.6, (b) 3.4 and (c, d) $3.6 \mathrm{~mm}$.

high concentration of PEGDA is therefore strong and brittle under elongation, whereas the network structure formed with low PEGDA concentrations should be more influenced by hydrogen bonds between cellulose fibers, and therefore, more conducive to elongation.

To determine the thermo-responsiveness of the BC/PEG-PEGDA gels prepared with $40 \%$ PEGDA, their optical transmission at a wavelength of $600 \mathrm{~nm}$ was measured every $5^{\circ} \mathrm{C}$. From the results presented in Figure 5, we see that unmodified BC/PEG gels exist as a white, opaque solid at low temperatures but become transparent at high temperatures. The point at which this physical change occurs is determined by the melting point of PEG. ${ }^{11}$ In a previous study, ${ }^{12}$ $\mathrm{BC} / \mathrm{PEG}$ gel was found to become transparent and elastic as the temperature increased above $25^{\circ} \mathrm{C}$, whereas the temperature at which BC/PEG-20\% PEGDA $(n=9)$ changed from opaque to $\sim 10 \%$ transparent varied between 25 and $30^{\circ} \mathrm{C}$. In this study, the change from opaque to $5-10 \%$ transparent still occurred at $25-30{ }^{\circ} \mathrm{C}$, indicating that the thermo-responsiveness of the BC/PEG-PEGDA gels slightly remains in comparison with the fully composite gel treated with 20\% PEGDA $(n=9)$. The decrease in transparency with increasing PEGDA concentration can be attributed to the aggregation of PEGDA on the fibers (Figure 1). Though the transmission of $\mathrm{BC} /$ PEG-40\% PEGDA for $24 \mathrm{~h}$ decreased, the transmission of BC/PEG$-40 \%$ PEGDA for $2 \mathrm{~h}$ was comparable to that of a fully composite BC gel formed with $20 \%$ PEGDA $(n=9) .{ }^{12}$ Thus, peripheral region crosslinking by PEGDA for $2 \mathrm{~h}$ is clearly effective in retaining the thermo-responsiveness of BC/PEG-PEGDA gels, as compared with the gels treated under other conditions.

\section{CONCLUSIONS}

Through this research, we have succeeded in developing BC/PEG gels that are nonbrittle under elongation by forming a composite with a crosslinked PEGDA network structure. These properties are achieved by limiting the depth to which the composite structure extends, thereby retaining a more ductile, fibrous phase in the center. Typically, materials become brittle with increasing hardness, but this study has demonstrated that a BC/PEG-PEGDA gel with a peripheral region crosslinked by PEGDA becomes both harder and less brittle than a $\mathrm{BC} / \mathrm{PEG}$ gel. Transmission measurements confirmed that the thermoresponsiveness of these $\mathrm{BC} / \mathrm{PEG}-\mathrm{PEGDA}$ gels is maintained as they change from opaque to transparent. These BC/PEG-PEGDA gels are 
also expected to be biocompatible, particularly given that PEGs are biocompatible and already extensively used in drug carriers, ${ }^{20}$ similar to PEGDAs. As BC and BC/PEG gels are typically considered too soft and difficult to handle for use as sheet materials, these findings suggest that BC/PEG-PEGDA gels have great potential for use in the fields of medicine and cosmetics for the treatment and repair of skin.

\section{CONFLICT OF INTEREST}

The authors declare no conflict of interest.

\section{ACKNOWLEDGEMENTS}

We thank Shin-Nakamura Chemical Co., Ltd for providing the PEGDA samples, Kenji Ohkubo and Mika Yamamoto of Hokkaido University for their help with the scanning electron microscope experiments and Honami Oono and Sakiko Nakamura of Otaru University of Commerce for their help with sample preparation. This study was supported by a Grant for Exploratory Research (AS24Z02456M) as part of the Adaptable and Seamless Technology Transfer Program through target-driven R\&D (A-STEP) of the Japan Science and Technology Agency (JST). A part of this work was conducted at Hokkaido University, supported by 'Nanotechnology Platform' Program of the Ministry of Education, Culture, Sports, Science and Technology (MEXT), Japan.

1 Iguchi, M., Yamanaka, S. \& Budhiono, A. Review. Bacterial cellulose - a masterpiece of nature's arts. J. Mater. Sci. 35, 261-270 (2000).

2 Ross, P., Mayer, R. \& Benziman, M. Cellulose biosynthesis and function in bacteria. Microbiol. Rev. 55, 35-58 (1991).

3 Brown Jr, R. M. The biosynthesis of cellulose. J. Macromol. Sci., A Pure Appl. Chem. 33, 1345-1373 (1996).

4 Okiyama, A., Motoki, M. \& Yamanaka, S. Bacterial cellulose III. Development of a new form of cellulose. Food Hydricilloid. 6, 493-501 (1993).
5 Klemm, D., Heublein, B., Fink, H. P. \& Bohn, A. Cellulose: fascinating biopolymer and sustainable raw material. Angew. Chem. Int. Ed. 44, 2-37 (2005).

6 Helenius, G., Backdahl, H., Bodin, A., Nannmark, U., Gatenholm, P. \& Risberg, B. In vivo biocompatibility of bacterial cellulose. J. Biomed. Mater. Res. A 76, 431-438 (2006).

7 Nakayama, A., Kakugo, A., Gong, J. P., Osada, Y., Takai, M., Erata, T. \& Kawano, S. High mechanical strength double-network hydrogel with bacterial cellulose. Adv. Funct. Mater. 14, 1124-1128 (2004).

8 Hagiwara, Y., Putra, A., Kakugo, A., Furukawa, H. \& Gong, J. P. Ligament-like tough double-network hydrogel based on bacterial cellulose. Cellulose 17, 93-101 (2010).

9 Fu, L., Zhang, J. \& Yang, G. Present status and applications of bacterial cellulose-based materials for skin tissue repair. Carbohydr. Polymers 92, 1432-1442 (2013).

10 Shah, N., UI-Islam, M., Khattak, W. A. \& Park, J. K. Overview of bacterial cellulose composites: a multipurpose advanced. Carbohydr. Polymers 98, 1585-1598 (2013).

11 Numata, Y., Muromoto, K., Furukawa, H., Gong, J. P., Tajima, K. \& Munekata, M. Nonvolatile and shape-memoried bacterial cellulose gels swollen by poly(ethylene glycol). Polymer J. 41, 524-525 (2009).

12 Numata, Y., Sakata, T., Furukawa, H. \& Tajima, K. Bacterial cellulose gels with high mechanical strength. Mater. Sci. Eng. C Mater. Biol. Appl. 47, 57-62 (2015).

13 Hestrin, S. \& Schramm, M. Synthesis of cellulose by Acetobacter xylinum. Biochem. J. 58, 345-352 (1954).

14 Yamanaka, S., Watanabe, K., Kitamura, N., Iguchi, M., Mitsuhashi, S., Nishi, Y. \& Uryu, M. The structure and mechanical properties of sheets prepared from bacterial cellulose. J. Mater. Sci. 24, 3141-3145 (1989).

15 Soykeabkaew, N. Sian, C., Gea, S., Nishino, T \& Peijs, T. All-cellulose nanocomposites by surface selective dissolution of bacterial cellulose. Cellulose 16, 435-444 (2009).

16 Gea, S., Bilotti, E., Reynolds, C. T., Soykeabkeaw, N. \& Peijs, T. Bacterial cellulose-poly (vinyl alcohol) nanocomposites prepared by an in-situ process. Mater. Lett. 64, 901-904 (2010).

17 Nishino, T. \& Arimoto, N. All-cellulose composite prepared by selective dissolving of fiber surface. Biomacromolecules 8, 2712-2716 (2007).

18 Soykeabkaew, N., Arimoto, N., Nishino, T. \& Peijs, T. All-cellulose composites by surface selective dissolution of aligned ligno-cellulosic fibres. Compos. Sci. Technol. 68, 2201-2207 (2008).

19 Soykeabkaew, N., Nishino, T. \& Peijs, T. All-cellulose composites of regenerated cellulose fibres by surface. Composites Part A 40, 321-328 (2009).

20 Kataoka, K., Harada, A. \& Nagasaki, Y. Block copolymer micelles for drug delivery: design, characterization and biological significance. Adv. Drug Deliv. Rev. 47 113-131 (2001).

Supplementary Information accompanies the paper on Polymer Journal website (http://www.nature.com/pj) 